\title{
La tarification des services d'électrification rurale décentralisée dans les pays en développement : méthode de calcul simplifiée et exemple d'application dans le cas de la petite hydroélectricité
}

\author{
Tariffs for rural electrification services in developing countries : \\ a simple methodology for calculation and its application \\ in the case of small hydropower
}

par Bernard Chabot

Expert senior, ADEME, Valbonne

Decentralised rural electrification is on the agenda of many countries and international organisations. In rural areas of developing countries, small hydropower plants are a first choice solution to electrify the villages which are too costly to connect to national or regional grids. This article proposes a simple general methodology to define the average tariff of decentralised rural electrification services from the profitability index of a reference project and its links with the average gross margin ratio of the rural electrification company. An example of an electrification of a typical village in a developing country based on a stand alone $25 \mathrm{~kW}$ small hydropower plant is described and discussed, including comparisons with an alternative solution based on the use of internal combustion engines. Three types of rural electrification companies with different management and marketing strategies are considered and the potential consequences of these differences on tariffs are presented and discussed.

\section{IINTRODUCTION}

L'électrification rurale décentralisée (ERD) intéresse de plus en plus les acteurs publics et privés du développement du fait de l'alternative qu'elle représente aux schémas d'électrification par extension des grands réseaux qui se révèlent incapables d'apporter rapidement le minimum de services énergétiques aux populations rurales des pays en développement n'ayant pas accès à l'électricité. Ces dernières représentent environ deux milliards d'habitants, ce nombre risquant malheureusement de diminuer trop lentement malgré les programmes d'électrification en cours et malgré l'exode rural, du fait de l'accroissement démographique important qui est prévu dans ces zones dans les deux décennies à venir.

Les solutions techniques et organisationnelles les mieux adaptées à l'ERD commencent à être connues et mises en pratique, que ce soit pour l'électrification par systèmes photovoltaïques sans réseau de distribution [1], ou pour l'électrification villageoise par un réseau autonome local alimenté par un petit diesel [2] ou une petite centrale hydroélectrique $(\mathrm{PCH})$. Dans ce dernier cas, la $\mathrm{PCH}$ est de très petite puissance (typiquement de 5 à $100 \mathrm{~kW}$ ) et le réseau local dessert dans un premier temps uniquement le village et ses alentours immédiats. Progressivement, ces PCH villageoises peuvent être amenées à être interconnectées entre elles et avec les réseaux régionaux et nationaux, comme cela est en cours en Chine où plus de 90 millions d'habitants ont été ainsi électrifiés de façon décentralisée depuis 1980 [3]. Dans toutes les solutions d'ERD, la nécessité de prendre en compte les principes de sobriété et d'efficacité énergétique au niveau des utilisateurs finaux est maintenant bien reconnue, ce qui permet de baser le choix des solutions d'électrification sur le coût du service énergétique final, mesuré par exemple par le Coût Global Actualisé du kWh Optimisê (CGAO) [4].

\section{II $\square$ COMPARAISON DES COÛTS}

Cette étape de connaissance des coûts du kWh optimisé de la solution optimale étant franchie, il reste à déterminer quel peut être le tarif de vente du service proposé, sous forme par exemple du tarif moyen du kWh optimisé. Bien sûr, il serait possible de compter dans les facteurs de coûts la rémunération financière visée par l'investisseur-opérateur, en choisissant pour le projet d'ERD considéré un taux d'actualisation 
égal au taux de rentabilité interne (TRI) espéré en menant l'opération à son terme. Par définition, la valeur actuelle nette (VAN) du projet est alors nulle, et le coût du $\mathrm{kWh}$ qui en résulte prenant déjà en compte le bénéfice financier recherché par l'investisseur, ce coût peut être considéré comme le tarif de vente (TV) à proposer aux utilisateursabonnés. Cette façon de procéder, très courante dans le monde anglo-saxon, présente cependant deux inconvénients majeurs :

- Elle entretient la confusion entre les notions de coût et de prix. Cela peut être dramatique quand cette démarche est basée sur le choix d'un taux d'actualisation (et donc dans ce cas d'un TRI) trop bas. Par exemple si ce TRI est pris simplement égal aux taux d'intérêt les plus favorables disponibles sur le marché financier, le résultat est que le tarif de vente sera égal au prix de revient du $\mathrm{kWh}$ sans bénéfice financier de l'investisseur-opérateur, ce qui ne pourra mener qu'à sa faillite à court terme.

- Les coûts d'investissements pris en compte pour un projet d'ERD ne comportent en général que des coûts directs : matières premières, achats et sous-traitances, main d'œuvre directe, frais d'études, de gestion et de supervision du projet, intérêts intercalaires. Or, une "société d'ERD" un tant soit peu ambitieuse et voulant passer par exemple en 10 ans «de mille à un million d'abonnés» devra couvrir des coûts énormes de croissance et de développement : R\&D, investissements de conception, de production et de gestion des équipements et des services, investissements commerciaux, frais généraux et frais financiers... Comme il est très difficile de répartir ces coûts projets par projets, le calcul du TRI de ces derniers ne pourra pas refléter leur rentabilité réelle.

Il est donc plus adéquat de considérer dès maintenant les opérateurs potentiels d'ERD comme des prestataires de services banalisés qui doivent se rémunérer en appliquant une marge sur le coût direct de leurs prestations (MCD) pour déterminer le tarif de vente (TV) de ces dernières.

Dans le cas de l'ERD, cette façon de faire présente des avantages certains :

- Pour l'analyse économique des projets d'ERD, elle permet de distinguer clairement le taux d'actualisation utilisé du TRI résultant de la réalisation dudit projet. Ainsi, les taux d'actualisation choisis peuvent-ils traduire sans ambiguïté soit la préférence pour les projets d'utilité sociale d'un opérateur d'ERD (par exemple un opérateur public), soit le coût moyen pondéré des ressources financières (fonds propres et emprunts) de cet opérateur.

- Outre la détermination toujours possible du TRI, critère préféré des directeurs financiers, cette façon de procéder permet aussi d'accéder aux critères d'analyse économique complémentaires plus utiles pour la stratégie industrielle d'un opérateur que ne le sont la Valeur Actuelle Nette réelle (VAN) et surtout le Taux d'Enrichissement en Capital (TEC, égal à la VAN dégagée par unité de capital investi) du projet.

- L'identification d'une marge sur les coûts directs (MCD) des prestations d'ERD permet de plus de comparer la gestion et les performances d'une société d'ERD avec celles de sociétés à activités voisines : compagnies d'électricité, prestataires de services en milieu rural, etc.

- La connaissance du coût direct du kWh d'ERD (son «coût Global Actualisé», CGA) et de la marge sur coût direct MCD choisie par l'opérateur permet d'accéder directement au tarif de vente TV du $\mathrm{kWh}$ optimisé final et de vérifier si ce tarif est compatible avec le pouvoir d'achat des abonnés potentiels d'un programme d'ERD. Si ce n'est pas le cas, outre la possibilité d'examiner si des réductions de coûts directs sont encore possibles, l'opérateur peut simuler l'impact d'une variation de sa marge moyenne traduisant des stratégies commerciales diversifiées : marge élevée et faible nombre d'abonnés ou marge faible et nombre élevé de clients.
Enfin, comme on le verra ci-dessous, pour des études de pré-faisabilité, la prise en compte d'un modèle simplifié d'exploitation d'un programme d'ERD permet d'accéder directement aux critères d'analyse économique recherchés par des formules simples et ne faisant intervenir que des ratios et permettant l'économie provisoire de «business plans» détaillés année par année et par postes de coûts et de recettes.

Ce modèle simplifié d'exploitation d'un programme d'ERD est basé sur l'hypothèse de cash-flows constants pendant la durée de vie économique du projet et l'hypothèse d'une inflation constante et d'une évolution des coûts et prix à un taux identique à celui de l'inflation. Il est décrit plus en détails, ainsi que les ratios et les formules pour déterminer le CGA du kWh, la VAN et le taux d'enrichissement en capital (TEC) du projet, dans la référence [5].

\section{APPLICATION À L'ÉLECTRIFICA- TION D'UN VILLAGE ISOLÉ}

Le projet type d'ERD qui va servir d'illustration est défini dans l'encadré 1 . Il concerne l'électrification d'un village isolé d'environ 1000 habitants (200 abonnés domestiques) par une petite centrale hydroélectrique $(\mathrm{PCH})$ permettant de couvrir des usages domestiques, de service public et d'activités économiques. Ce projet de référence intègre au maximum les appareils et procédés à haute efficacité énergétique (par exemple éclairage par fluorescence). La place importante des usages productifs ( $1 / 3$ de l'énergie distribuée) permet par ailleurs un facteur de charge correct de la centrale, tout en favorisant le renforcement du développement économique en cours, garant d'un minimum de capacité à payer les services domestiques, les services marchands et les services communautaires. Dans cet exemple type, compte tenu du niveau des besoins énergétiques optimisés finaux ( 250 $\mathrm{kWh} / \mathrm{jour})$, de l'isolement et de la compacité de ce village (seulement $4 \mathrm{~km}$ de réseau de distribution BT simplifié et à faible contenu d'importation), l'alternative à la $\mathrm{PCH}$ ne serait ni le photovoltaïque ni une extension de réseau, mais l'usage de groupes électrogènes privés, dont le coût de production du $\mathrm{kWh}$ serait de l'ordre de 0,37 Euro/kWh ( $\left.{ }^{1}\right)$, comme on peut le voir dans l'encadré 1 .

Les définitions, les ratios et les formules de base utilisées pour cette analyse simplifiée sont rappelées dans l'encadré 2. Elles permettent notamment de calculer le CGA du $\mathrm{kWh}$ produit et de déterminer les paramètres ci-dessous en fonction de la MCD choisie par l'opérateur (ici trois variantes : $\mathrm{MCD}=25 \%, 33,3 \%$ ou $50 \%$ ):

- Le tarif de vente moyen du kWh: TV.

- Le TEC du projet (et donc aussi la VAN du projet qui est égale à TEC fois l'investissement initial $I$ ).

L'analyse porte de plus sur trois cas différents de réalisation et d'exploitation du projet d'ERD :

- Cas $\mathbf{N}^{\circ} 1$ : Investisseur/exploitant privé, se finançant sur le marché financier local (d'où un choix de taux d'actualisation réel $\left({ }^{2}\right) t=12 \%$ ) et cherchant un TRI élevé, supérieur ou égal aux opportunités de rentabilité les meilleures en $\mathrm{PED}$, soit typiquement 20 à $40 \%$. Cet opérateur considère par ailleurs que l'ERD risque d'être une solution d'attente de l'électrification par extension de réseau, ce qui l'incite encore plus à baser son analyse économique sur une durée d'observation faible ( $n=10$ ans).

(1) 1 Euro $=6,55957 \mathrm{FRF}=1,02$ US $\$$

$\left({ }^{2}\right)$ Le taux d'actualisation réel $t$ se déduit du taux nominal $t n$ et du taux d'inflation $i$ (supposé constant) par la relation : $(1+t)=(1+t m) /(1+i)$, qui peut se simplifier si $i<0,1(10 \%)$ en : $t=t n-i$. 


\section{ENCADRÉ 1. DESCRIPTION DU PROJET D'ERD DE RÉFÉRENCE}

\section{1) Le village :}

Il s'agit d'un village de 1000 habitants (200 familles) en bord de rivière pérenne, connaissant un début de développement économique soutenu (agriculture, artisanat), trop loin du réseau pour envisager une interconnexion. Il s'agit d'un habitat groupé, rendant possible une distribution par réseau BT simplifié.

2) Les besoins :

- Besoins domestiques moyens : $750 \mathrm{Wh} / \mathrm{j}$.abonné (éclairage, radio K7, TV, $10 \%$ des ménages susceptibles de s’équiper en réfrigérateur/conservateur), occasionnellement petit électroménager et petit outillage, cuiseur et bouilloire électrique de faible puissance pour valorisation des surplus d'électricité en heures creuses. Total des consommations domestiques: 150 $\mathrm{kWh} /$ jour.

- Besoins communautaires : pompe d'adduction d'eau $6 \mathrm{kWh} / \mathrm{j}\left(40 \mathrm{~m}^{3} / \mathrm{j}\right.$ à $20 \mathrm{~m}$ de $\left.\mathrm{Hmt}\right)$; éclairage public : 3,2 kWh/j ; bâtiments publics (école, maison commune, centre religieux) : $3 \mathrm{kWh} / \mathrm{j}$.

- Services privés : trois commerces $(3 \mathrm{kWh} / \mathrm{j})$.

- Besoins productifs : atelier/meunerie : $28 \mathrm{kWh} / \mathrm{j}$; irrigation/drainage : $10000 \mathrm{~m} / \mathrm{j}$, soit $56 \mathrm{kWh} / \mathrm{j}$.

- Total : $250 \mathrm{kWh} / \mathrm{j}$; production correspondante avec un rendement de distribution de $92 \%: 272 \mathrm{kWh} / \mathrm{j}(100000$ $\mathrm{kWh} / \mathrm{an})$.

3) Le système électrique :

- PCH de $25 \mathrm{~kW}$. Coût installé : $75 \mathrm{kEuro}(I u=3000$ Euro/kW). Facteur de charge : $N h=4000$ heures équivalentes par an de fonctionnement à puissance nominale.

- Réseau de distribution allégé : longueur développée : $4 \mathrm{~km}$. Coût : 75 kEuro (y compris 150 Euro de branchement par abonné).

- Coût total : $150 \mathrm{kEuro,} \mathrm{soit} I u=6000 \mathrm{Euro} / \mathrm{kW}$ installé.

4) Les alternatives :

- Photovoltaïque : mal adapté dans ce cas compte tenu de l'importance des consommations : la puissance crête requise (base gisement solaire de $5 \mathrm{kWh} / \mathrm{m}^{2} . \mathrm{j}$ ) serait d'environ $84 \mathrm{kWc}$ (coût d'investissement minimum : $1000 \mathrm{kEuro}$ ).

- Groupe électrogène diesel privé, coût unitaire d'investissement de 1000 Euro/kW, facteur de charge $N h=3000 \mathrm{~h} / \mathrm{an}$, rendement global $15 \%$, coût de combustible 0,5 Euro/l, soit en actualisant à $12 \%$ sur 10 ans, un coût de production du $\mathrm{kWh}$ de 0,366 Euro/kWh.

\section{ENCADRÉ 2. FORMULES DE BASE POUR L'ANALYSE ÉCONOMIQUE SIMPLIFIÉE}

\section{1) Définitions et ratios :}

$N h:$ (heures/an ou $\mathrm{kWh} / \mathrm{kW}$ - an) : nombre d'heures de fonctionnement équivalent du générateur d'électricité (de puissance nominale $P \mathrm{~kW}$ et produisant $E a \mathrm{kWh} / \mathrm{an})$ à sa puissance nominale $: N h=E a / P$.

$I$ (Euro) : investissement total (études, fournitures, aménagements du site, installation, coûts de gestion et de supervision du projet, intérêts intercalaires...).

$I u($ Euro $/ \mathrm{kW}):$ investissement unitaire $: I u=I / P$.

$K a$ : coefficient d'actualisation : $K a=t(1+t)^{n} /\left((1+t)^{n}-1\right)$, avec $t=$ taux d'actualisation réel et $n=$ durée d'observation.

Dem (Euro/an) : dépenses annuelles moyennées d'exploitation et d'entretien-maintenance.

$\mathrm{Kem}$ : coefficient d'exploitation et d'entretien-maintenance : $\mathrm{Kem}=\mathrm{Dem} / \mathrm{I}$.

$X:$ Coefficient multiplicateur $=\mathrm{TV} / \mathrm{CGA}=$ tarif de vente moyen du $\mathrm{kWh} /$ Coût Global Actualisé du $\mathrm{kWh}$.

$M C D$ : Marge sur Coût Direct $=($ TV-CGA $) / T V$; relation entre $X$ et MCD : $X=1 /(1-\mathrm{MCD})$.

VAN : Valeur Actuelle Nette d'un projet de coût d'investissement initial $I$ sur une durée d'observation de $n$ années avec un taux d'actualisation réel $t$ :

$\operatorname{VAN}(I, t, n)=-I+\sum_{j=1}$ à n $\left((\right.$ Encaissementş-Décaissements $\left.) /(1+t)^{j}\right)$

TRI : Taux de Rentabilité Interne d'un projet $=$ valeur du taux d'actualisation $t$ annulant $\operatorname{VAN}(I, t, n)$.

2) Calcul du CGA (coût Global Actualisé) du kWh :

$C G A=($ somme des dépenses actualisées)/(somme des $\mathrm{kWh}$ actualisés), soit pour une centrale sans coûts de combustible (solaire, $\mathrm{PCH}$, éolien) :

$C G A=I u(K a+K e m) / N h$

\section{3) Calcul du Taux d'Enrichissement en Capital TEC :}

Par définition TEC $=$ VAN générée par unité de capital investi $=$ VAN $(I, t, n) / I$.

Pour un projet avec des cash-flow (différence entre les encaissements et les décaissements) constants et une centrale sans coûts de combustible :

$T E C=T V \cdot N h /(K a \cdot I u)-(1+K e m / K a)$

et on peut déduire le TEC d'un projet à partir de $\mathrm{MCD}$ par les relations suivantes :

$X=T V / C G A=1 /(1-M C D)=1+(K a /(K a+K e m)) \cdot T E C$ 
Tableau 1. - Résultats de I'analyse du projet ERD de I'encadré 1.

\begin{tabular}{|c|c|c|c|c|}
\hline \multicolumn{2}{|c|}{$\begin{array}{c}\text { Marge sur Coût Direct MCD }=(\text { TV-CGA }) / T V(\%) \\
\text { Coefficient multiplicateur } X=T V / C G A=1 /(1-M C D)\end{array}$} & $\begin{array}{l}25 \\
1,33\end{array}$ & $\begin{array}{r}33,3 \\
1,5\end{array}$ & $\begin{array}{r}50 \\
2\end{array}$ \\
\hline Cas $\mathbf{N}^{*} 1$ & Tarif de vente TV (Euro/kWh) & 0,434 & 0,488 & 0,651 \\
\hline$t=12 \%$ & $\mathrm{TEC}=\mathrm{VAN} / \mathrm{I}$ & 0,41 & 0,61 & 1,23 \\
\hline$n=10$ ans & VAN (kEuro) & 61,3 & 91,8 & 183,9 \\
\hline CGA $=0,325$ Euro $/ \mathrm{kWh}$ & TRI $(\%)$ & 21,3 & 25,6 & 37,7 \\
\hline Cas $\mathbf{N}^{*} \mathbf{2}$ & Tarif de vente TV (Euro/kWh) & 0,314 & 0,353 & 0,470 \\
\hline$t=8 \%$ & $\mathrm{TEC}=\mathrm{VAN} / \mathrm{I}$ & 0,45 & 0,67 & 1,34 \\
\hline$n=15$ ans & VAN (kEuro) & 67,1 & 100,5 & 201,4 \\
\hline $\mathrm{CGA}=0,235$ Euro $/ \mathrm{kWh}$ & TRI (\%) & 14,8 & 17,9 & 26,6 \\
\hline Cas $\mathbf{N}^{\bullet} \mathbf{3}$ & Tarif de vente TV (Euro/kWh) & 0,182 & 0,205 & 0,273 \\
\hline$t=3 \%$ & $\mathrm{TEC}=\mathrm{VAN} / \mathrm{I}$ & 0,59 & 0,89 & 1,78 \\
\hline$n=30$ ans & VAN (kEuro) & 89,2 & 133,6 & 267,6 \\
\hline $\mathrm{CGA}=0,137$ Euro $/ \mathrm{kWh}$ & TRI (\%) & 7,1 & 8,9 & 13,9 \\
\hline
\end{tabular}

- Cas $\mathbf{N}^{\circ} 2$ : Investisseur/exploitant privé, voulant s'investir sur le long terme sur l'ERD et en devenir un acteur majeur au niveau mondial afin d'avoir accès au moins partiellement à des financements internationaux plus favorables que les financements locaux et utilisant donc un taux d'actualisation moins élevé ( $t=8 \%$ réels), sur une durée d'observation économique plus longue ( $n=15$ ans).

- Cas $\mathbf{N}^{\circ} 3$ : opérateur public (agence nationale d'électrification rurale) ou para-public (collectivité territoriale via une société d'économie mixte, filiale ERD d'une compagnie d'électricité nationalisée ayant obtenu une «liberté encadrée» pour fixer ses tarifs de vente du $\mathrm{kWh}$ en zones rurales iso- lées...) et incitée par ses autorités de tutelle à utiliser des conditions d'actualisation ( $t=3 \%$ réels, $n=30$ ans) favorisant les investissements d'utilité sociale à long terme et éligibles à des prêts bonifiés internationaux.

Les résultats de cette analyse sont résumés dans le tableau 1 et les figures 1 et 2 . On en déduit en particulier les observations et conclusions suivantes :

- Les tarifs de vente TV du kWh sont plus élevés que les tarifs péréqués de l'électricité sur grands réseaux interconnectés. En zones rurales, ces tarifs péréqués étant en général largement en dessous des coûts réels de production, de transport et de distribution, il ne faudrait bien sûr pas en

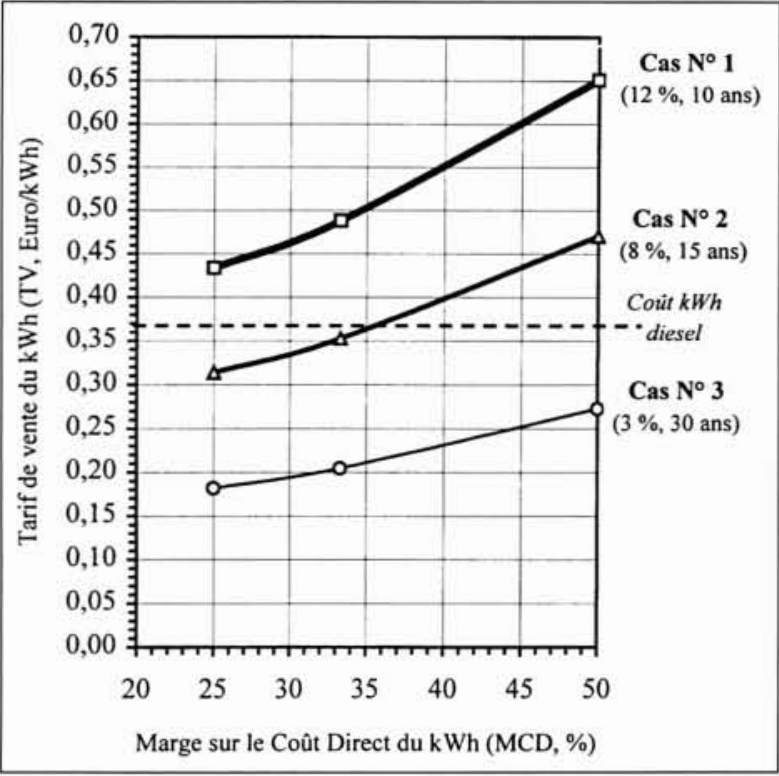

1. Prix de vente du $\mathrm{kWh}$ en fonction de la marge sur coûts directs et des conditions d'actualisation.

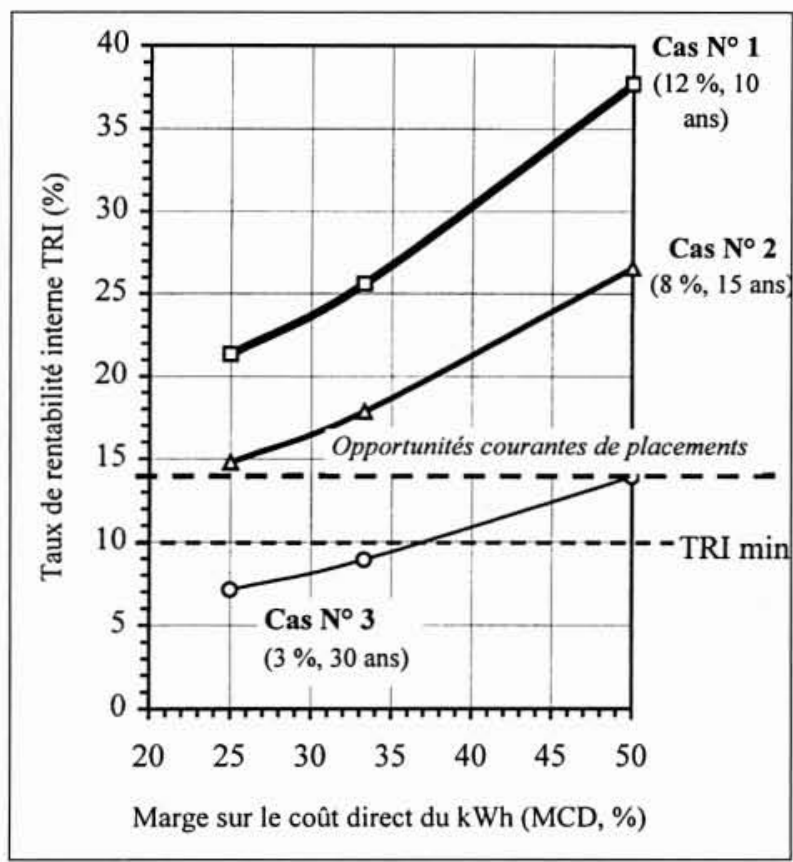

2. TRI en fonction de la marge sur coût direct et des conditions d'actualisation. 
déduire que l'ERD n'est pas «rentable». Au contraire il faut en déduire qu'il faut donner aux exploitants d'ERD la liberté de fixer leurs tarifs en fonction des coûts réels des services correspondants, dans le cadre d'une régulation assurant un minimum de concurrence et d'engagements sur l'équité, la pérennité et la qualité du service global d'ERD.

- Les tarifs sont extrêmement variables en fonction des cas étudiés et des valeurs extrêmes des marges sur coûts directs (de 0,182 à 0,651 Euro/kWh). Cela reflète la diversité des stratégies organisationnelles et commerciales que peuvent choisir les opérateurs de l'ERD.

- Dans le cas $\mathbf{N}^{\circ} \mathbf{1}$, comme on le voit en figure 1 , le tarif de vente du $\mathrm{kWh}$ est toujours supérieur au coût de référence du groupe électrogène diesel ; il y a donc un risque évident que des clients potentiels (notamment ceux ayant des activités artisanales ou commerciales) refusent de s'abonner et préfèrent s'équiper directement en groupes électrogènes individuels. Le marché de l'ERD pour les investisseurs/exploitants correspondant au cas $\mathrm{N}^{\circ} 1$ devrait donc être très étroit et limité à des projets où la concurrence serait quasiment absente. En clair, ce cas $\mathrm{N}^{\circ} 1$ ne peut pas donner lieu à des modèles ou des stratégies réalistes et viables d'ERD.

- Dans le cas $\mathbf{N}^{\circ} \mathbf{2}$, on voit en figure 1 que la MCD doit être inférieure à $35 \%$ pour que le tarif de vente proposé soit inférieur au coût de référence d'un groupe électrogène individuel. Cette limite est sans doute compatible avec un développement pérenne de l'entreprise d'ERD, sous réserve d'une gestion performante (limitation des coûts indirects et des frais généraux, politique commerciale contribuant à faire croître rapidement le nombre d'abonnés à la société d'ERD dans le pays ou les zones géographiques où elle compte intervenir...). On voit en figure 2 que les TRI correspondant à cette limitation de la MCD sont supérieurs aux opportunités courantes de placements financiers dans les PED telles que citées par exemple dans la référence [6], soit environ $14 \%$.

- Dans le cas $\mathbf{N}^{\circ} \mathbf{3}$, on voit en figure 1 que le tarif de vente est toujours inférieur au coût de référence du groupe électrogène individuel, mais on voit en figure 2 que seules des MCD supérieures ou égales à $37 \%$ permettent des TRI supérieurs à la limite inférieure préconisée en référence [6] pour des projets en PED, soit $10 \%$. Le tarif de vente TV correspondant à une MCD de $37 \%$ (cf. fig. 1) serait alors de 0,225 Euro/kWh, soit seulement $64 \%$ du tarif du cas $\mathrm{N}^{\circ} 2$ avec une MCD de $35 \%$. Dans le cas où un TRI au moins égal aux opportunités courantes de placements serait recherché, on voit en figure 2 que la MCD devrait être d'au moins $50 \%$, ce qui mènerait à un tarif de 0,273 Euro $/ \mathrm{kWh}$, encore inférieur aux tarifs envisageables dans le cas $\mathrm{N}^{\circ} 2$. L'implication des pouvoirs publics et la mobilisation de financements internationaux à taux bonifiés mènent donc, comme cela était prévisible, à des tarifs plus attractifs pour les clients, même lorsque l'entreprise d'ERD pratique des marges élevées. Ces conditions permettant de créer un cadre favorable à l'ERD sont donc à recommander chaque fois qu'elles sont possibles.

\section{IV $\square$ CONCLUSION}

Les conclusions tirées de l'exemple ci-dessus et des différents cas d'investissement et d'exploitation ne sont bien sûr pas absolues et ne couvrent pas tout le champ des cas possibles. Notamment, l'accès d'opérateurs privés du type de ceux envisagés dans le cas $\mathrm{N}^{\circ} 2$ aux financement à taux bonifiés devrait être de plus en plus courant à l'avenir, ce qui permettrait à ces opérateurs de prendre en compte des taux d'actualisation moins élevés.
Après cette première analyse économique avec le modèle et les outils simplifiés présentés ici, et qui permet de cerner les principales options à retenir pour un avant-projet au niveau d'une étude de pré-faisabilité, il faudra procéder à une analyse financière détaillée faisant intervenir le partage entre les fonds propres et les emprunts pour le financement des projets ainsi que l'impact de la fiscalité et des subventions éventuelles. En effet, comme dans le cas de l'électrification classique par extension des réseaux interconnectés, il peut être opportun de consacrer des subventions à la diffusion de l'ERD, soit directement par l'Etat, soit indirectement via des subventions croisées entre les consommateurs urbains et ruraux. Une telle analyse détaillée, qui dépasserait largement le cadre de cet article, doit aussi intégrer la dynamique des projets, année par année, afin de prendre en compte par exemple l'évolution dans le temps du nombre d'abonnés et une évolution éventuelle des coûts et des tarifs.

Compte tenu des incertitudes inhérentes au devenir réel à long terme des programmes d'ERD en cours ou projetés, les hypothèses simplificatrices sur lesquelles est basée cette proposition d'analyse économique simplifiée des avant-projets d'ERD à partir des marges sur le coût direct du kWh garde tout son intérêt et toute sa pertinence pour procéder au choix des options principales qui se présentent aux promoteurs et aux opérateurs de l'ERD. Enfin, la méthode correspondante peut être aisément transposée au cas des autres filières énergétiques et au cas des services énergétiques finaux non électriques, comme le cas de la vente d'eau en zones rurales à partir de pompes solaires photovoltaïques [7].

Il reste cependant à tester cette nouvelle méthode simplifiée d'analyse économique sur des cas réels, afin de mieux cerner sa précision et sa pertinence par rapport aux analyses financières détaillées. Cela permettrait en particulier de déterminer les valeurs minimales des marges sur coûts directs à préconiser pour assurer un développement pérenne aux acteurs de l'ERD.

\section{RÉFÉRENCES}

[1] LoRenzo E. - «Photovoltaic rural electrification». Prog. Photovolt. Res. Appl., vol. 5, pp. 3-27, 1997. Cet article de synthèse contient une bibliographie avec 113 références sur le sujet.

[2] ThiвоN J. - «Le concept GECO, un principe d'électrification rurale décentralisée adapté au contexte socioéconomique Africain». Liaison Energie-Francophonie, $\mathrm{N}^{*} 26$, 1995, Québec.

[3] Jiandong T. - «Small Hydro Power: China's Practice». Hangzhou Regional Center Publications, 1994, Hangzhou.

[4] Снавот B. - «Electrification rurale : méthodes et outils d'analyse». Systèmes Solaires, $N^{\circ} 120,1997$, Paris.

[5] Снавот B. - Chapitre 4: "Analyse économique» du "Guide de I'Energie Eolienne». FONDEM/IEPF, 1998, Paris.

[6] Goldsmith K. - «The Role of the Private Sector in the Small-Scale Hydropower Field». MHPG Series Harnessing Water Power on a Small Scale, Vol. 12, SKAT, St Gallen, 1995.

[7] Снавот B. - «From Costs to Prices: Economic Analysis of Photovoltaic Energy and Services». Progress in Photovoltaics : Research and Applications, vol. 6, pp. 55-68, John Wiley \& sons, 1998. 